\title{
Media Faktura. On some Technical Conditions of Image-Making in Art
}

Andreas Broeckmann,

Leuphana University of Lüneburg

\begin{abstract}
The text contributes to the discussion about the ontological status of the "image" by offering an analysis of the technical and material conditions of image-making. Departing from a close reading of French artist Julien Maire's installation Memory Cone (2009), the paper discusses four distinct types of technical conditions which determine mediated images: physiological, physical, electronic, and algorithmic. It references art historical examples to argue that such technical conditions have always been fundamental to images, and suggests the interdependency between these medial layers.
\end{abstract}

Keywords: art, image theory, media specificity, technology, materiality

\section{INTRODUCTION}

The materiality and mediality of artworks have-from Constructivism, through Abstract Expressionism, to Conceptualism-been at the center of modern conceptions of art. At the same time, the ontological and the epistemological status of the image, and of visual representations in general, has been the topic of discussions in the humanities and the sciences. They have recently been condensed in the debates about the "pictorial" and "iconic turns", and about Visual Studies or, in the German-speaking world, „Bildwissenschaft.“ Here attempts are made to describe the relation between art-historical and art-theoretical conceptions of the image and its status in scientific imaging, data visualization, and computer simulations. The intentionality of an image and the conditions under which it is conceived and made are seen as crucial for its aesthetical and epistemological status.

These issues are pinpointed by artworks in which images are created by machines. From the introduction of photography onwards, the mechanized production of images has been a challenge for the pictorial arts, and for the definition of how visual evidence is constructed. 'The installations and projection apparatuses of the French artist Julien Maire address crucial aspects of this discussion, because they help to broaden our understanding of what constitutes an „image“ under contemporary technological conditions. In order to develop an analytical toolbox for studying images created by mechanical, electronic and digital apparatuses, this paper proposes to distinguish four different types of artistic "image machines," which can be identified in examples from throughout the 20th century: optico-physiological image machines, mechanical image machines, media-technical apparatuses, and algorithmic image machines. They each construct images under different conditions and thus build different relations between image, medium, and viewer.

The "image machines" discussed here are apparatuses created and used not as mere playback devices, but as artistic machines which bring forth the images that we see, and which are recognizable as partly autonomous actors whose aesthetic output is determined not by the human operator of a technical medium, but by the configuration of the "machinic" technical installation. Many of these image machines have the status of an artwork, either because of their sculptural form, or due to the kinetic, electronic or other processes that they engender. What, then, would typical "machine images" be, and how do "image machines" push the notion not only of "the image", but also of the perceiving subject that they construct? ${ }^{2}$

\section{JULIEN MAIRE'S "MEMORY CONE"}

Julien Maire is a French artist who explores the correlation of image, medium and memory in his deconstructive, media-archaeological installations and performances. His project "Memory Cone" (2009) is an interactive installation whose apparatus 


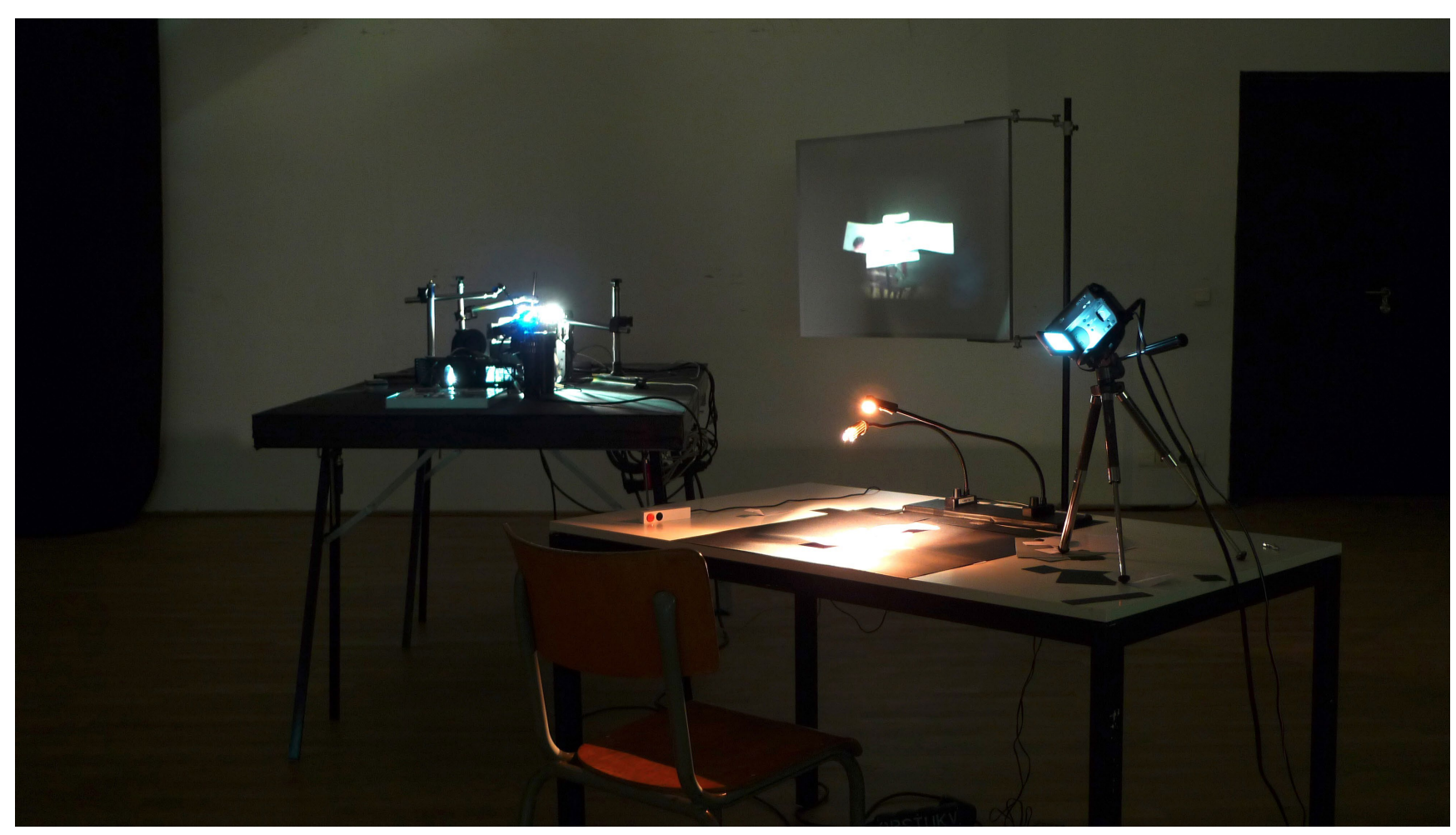

Julien Maire: Memory Cone (2009), installation view, MUHKA, Antwerp.

produces still and fragmented images from found slides, in a hybrid interaction of human, optical, mechanical, and electronic agents.

The exhibition visitor encounters a table with a black table cloth. There is a video camera on a small tripod pointed at the black cloth, and, on a separate table and connected to the camera, a video projector with unusual extensions, that projects onto a white screen. White strips of paper can be placed by the visitor on the black cloth, and as they are moved into the field of view of the video camera, the projector displays equivalent parts of what look like generic family images. Thus, rectangle or square fragment parts of an image can be scanned and explored by moving around the white paper, or by placing additional strips which will enlarge the zone of visibility of the image. Gaps between the papers, and a shadow that one piece may throw on another, show up on the projection screen as empty black, as the type of void that can be generalized-and the image removed entirely - at a swipe of the hand.

The production of the projected image remains enigmatic, for although the entire apparatus appears in front of our eyes, it is difficult to understand where the image is actually generated. The video camera is only pointed at the table top, recording the differences between black and white. Part of the extended video projector is a straight-tray slide projector with slides that are consecutively projected.

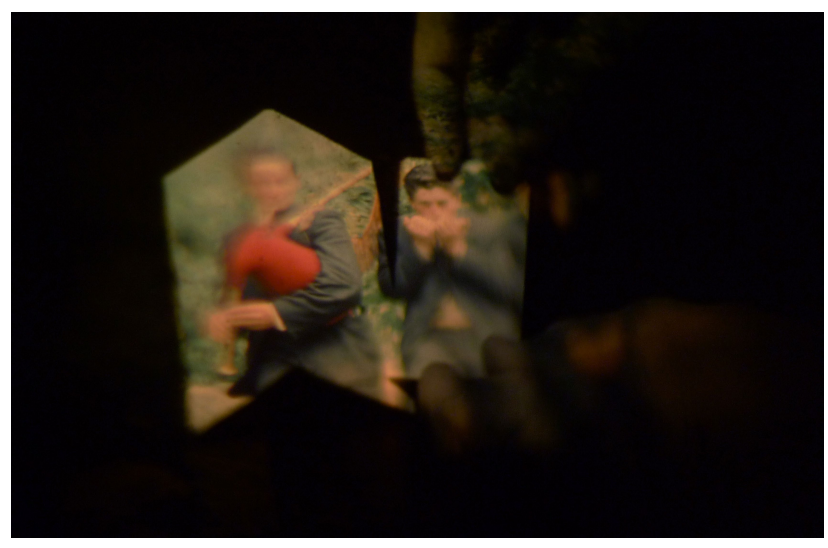

Julien Maire: Memory Cone (2009), projection.

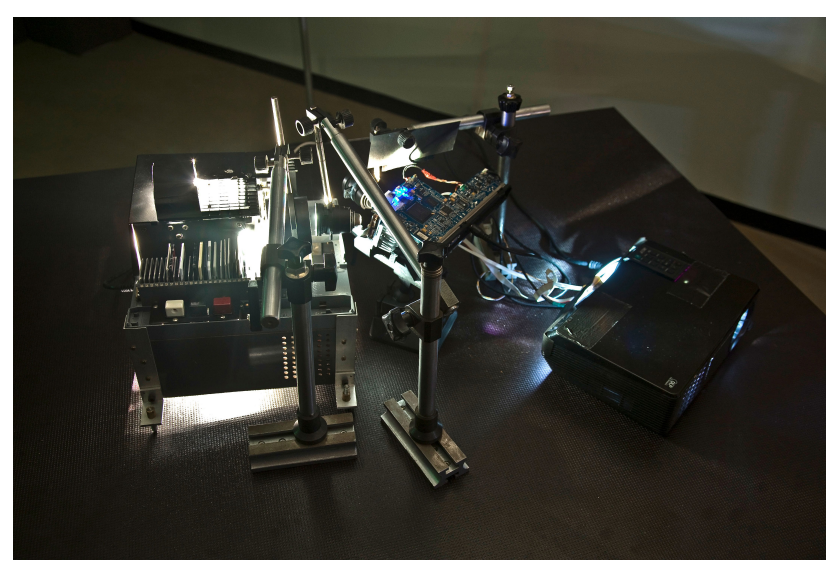

Julien Maire: Memory Cone (2009), projection apparatus. 
A special set of optical lenses reduces the image from this slide projector and directs it at a mirror device that is part of the DLP (Digital Light Processing) technology used in contemporary video projectors. This DMD (Digital Micromirror Device) carries hundreds of thousands of small mirrors on its ca. $2 \times 3.5$ $\mathrm{cm}$ surface. Each of these small mirrors can be tilted electronically to produce, depending on the speed at which it is tilted back and forth, multiple shades of gray. In a video projector using the DLP technology, the DMD together with a special color wheel technology can produce color video images of HD quality, using a white light source.

Julien Maire repurposes this technology in the „Memory Cone“ (2009) installation: his DLP video projector regularly converts the live image taken by the video camera on the table top into the projection of an, in this case, dark gray surface image. When a white strip is moved into the field of view of the camera, the respective micro mirrors on the DMD are tilted at a frequency that normally produces the equivalent of brightness. The color wheel of the projector is by-passed, so the image of the table top, and of the hands arranging the white paper strips, is without technical color manipulation. The light source, however, is not a regular white LED, but the slide projector whose special optical lens reduces and points the slide image at the DMD. The light that gets projected onto the screen is the light from the slide projector bulb, filtered by the slide, so that all the colors, the bright and the dark areas of the slide determine the composition of the image triggered by the white strips. This effect can also be observed when a hand reaches into the field of view of the camera: the visitor's hand then becomes the light trigger and defines, with its shape and areas of brightness and dark, the shape of the parts of the slide image projected onto the screen. While

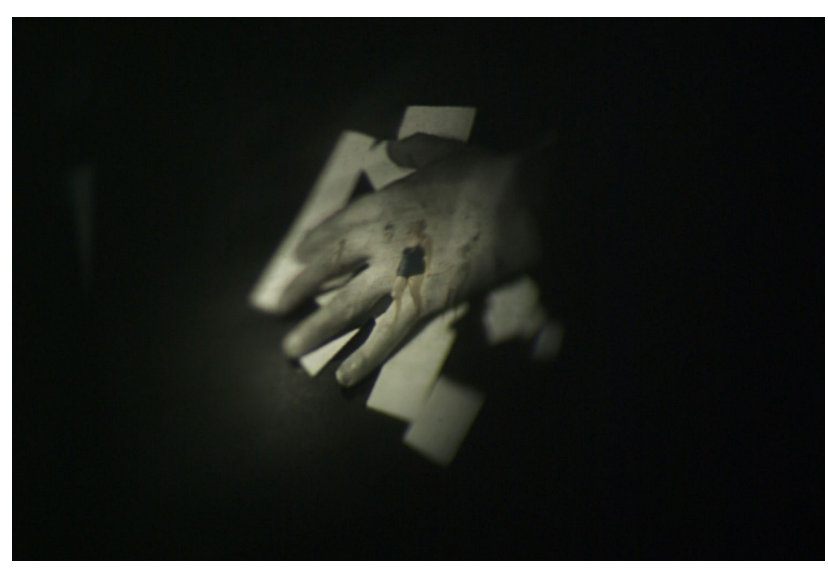

Julien Maire: Memory Cone (2009), projection. the slide image is reversed by the DMD mirrors, the shaping video image is, under normal circumstances, a straight, analogical presentation of the camera image-although, of course, the settings in either the camera or the projector could easily be changed to a reversed version of the camera image.

This complex arrangement raises the question of what the image is, and where it actually occurs. The Belgian film and media historian Edwin Carels has suggested that, "the status of the image in Memory Cone can be described neither as a photograph nor as a slide, a video still or a film still. A video image without pixels? A quietly vibrating photograph?" ${ }^{3}$ But it is also possible to interpret Maire's proposal differently and argue that, on the contrary, it is possible to precisely describe the image in its different stages by considering these stages not as exclusive alternatives ("or"), but as states that can be hybridized and accumulated (" and "). What we see on the wall is an analogue reflection of the light that is projected by the slide projector onto the DMD mirror device. The micro-mirrors are positioned according to the images taken continuously by the video camera of the table top, and electronically processed according to the DLP protocols. The physical interaction of the visitor on the white strips is electronically mediated by the video camera and translated into the physical movements of the micro mirrors on the DMD chip. What becomes visible of the photographic (positive) image projected by the slide projector is technically conditioned by the electronically controlled configuration of the DMD mirrors, and visually conditioned by the placement of the white strips by the visitor.

\section{TECHNICAL CONDITIONS OF IMAGE-MAKING}

The aesthetics of such images hinges on a variety of aspects, one of which is the apparent automatism which brings about and transforms the images. Each media-technical dispositive (M. Foucault) offers a variety of points where such automatisms can occur. In Julien Maire's Memory Cone, it is difficult to speak of "an image“, when actually there is a whole chain of material, visual and electronic events, each of which can be addressed as an image, or as an image source, and each of which is taken, processed and passed on to other parts of the technical assemblage. The two main image sources are coupled optically (the slide projector) and electronically (the video camera) to produce one projected image that is an optical mirror reflection of the slide image, with the light configuration being shaped by the digitally processed, electronic transmission of the camera image onto the DMD. 
In an insightful discussion about the principle relation between image and medium, Gottfried Boehm has suggested, as a minimal definition of image, "the simultaneous perceivability of the representation and the represented, of the medial premise and the iconic shaping." ${ }^{\text {B }}$ Boehm claims that certain media (like stained glass, or the principles of perspective) imply certain aesthetics, and that the apparatuses that serve to mechanically support the construction of central perspective (like the velum, the camera obscura, or the photo camera) imply an inferior aesthetics of reproduction. ${ }^{5}$ According to Boehm, such inferiority can be overcome to the degree that the images transgress the mediality of the conditions that frame them. ${ }^{6}$

What we are interested in here are the technical conditions of image-making, and the aesthetic automatisms that appear to go along with them. We always look at images under specific material, technical and physiological conditions, and the specific technical conditions under which images exist always contain the potential of automatisms. The four types of such conditions that we will look at more closely are the physiological, physical, electronic, and algorithmic determinants of images. These are not only the material conditions of images and their perception, but each of these clusters of conditions also brings with it a certain level of aesthetic latitude, a range of potentials that determine what is perceived as an image beyond the inscriptions of human will, or artistic intention. In so far as the apparatuses and apparitions of images before us are beyond such intention, their dispositives can be conceived as image-machines which produce images beyond human intervention, images whose aesthetics hinges on their production by such machines.

\subsection{Physiological Conditions of Image-Making} Although physiologically grounded visual phenomena like the after-image had been known since antiquity, it was not before the 19th century that they were systematically researched and described. In his seminal study on the "Techniques of the Observer" (1990), Jonathan Crary discussed the parallel developments of this research and of the media techniques that made it possible to study the respective phenomena, and that turned some of these phenomena into entertaining spectacles. ${ }^{7}$ Crucially, the researchers of the 19th century discovered the physiologically determined latency, and thus the temporality of seeing, and hence the autonomy of the act of seeing, from the seen object. The eye and the human perceptual (optical and neurological) apparatus was conceived as part of a continuum that, by means of such media techniques as stereoscopy or stroboscopy, and under specifically controlled spatial and lighting conditions, could be made to perceive images as moving or three-dimensional. These perceptions are effects of the respective media apparatus - as all images are.

The "representation of the representation" (Boehm) has been exercised and transgressed by artists throughout modernity-the paintings of Paul Cézanne are the classic illustration of this trope. The "representation of perception" and of the mediality of human perception was epitomized by Marcel Duchamp's Roto-Reliefs of the 1920s and 1930s. Duchamp produced six double-sided disks of $20 \mathrm{~cm}$ diameter which were to be rotated at a speed of 40-60 rpm by a gramophone-like motorized apparatus. When spun like that, the designs on the disks, entitled for instance, "Montgolfière“, "Gold Fish“, or "Chinese Lampion", appear to the human eye as though parts of the designs were making additional, or contrary movements, or as though certain elements were three-dimensional. ${ }^{8}$

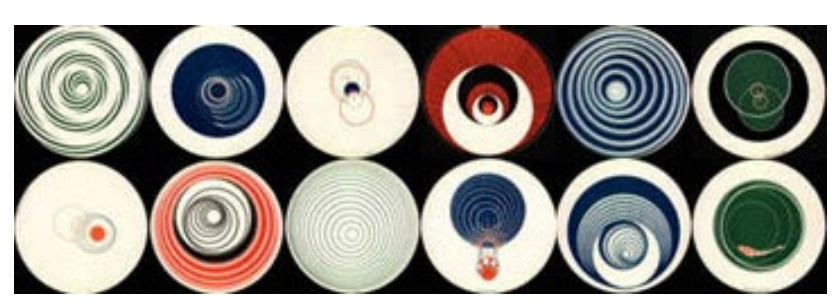

Marcel Duchamp: Roto-Reliefs (1935), disks.

The Roto-Reliefs form part of Duchamp's critique of a retinal art which reproduces visual experiences. Instead, he sought to enable a new type of experience that directly speaks to the mind. Yet, at the same time, the spinning disks can be understood as "hyper-retinal", in that they allow the experience of observing at least part of the processes through which the human visual apparatus creates the impression of movement and spatial depth from specific graphic and dynamic configurations.

What appears as an image of the spinning RotoReliefs is a phenomenon which uniquely and exclusively appears to the human eye. This phenomenon is latent in the design printed on the disk, and will be realized only under the conditions of the motor spinning the disk at a particular speed, and the physiological conditions of the perceiving eye.

Research into the functioning of human vision led, around 1830 , to the discovery of the stroboscopic effect: based on the latency of the after-image, images that appear to the human eye in discrete form will be perceived as a continuous, moving sequence, if the differences between the individual images are small enough. The phenakistiscope and the zoetrope 
were the first devices that made use of this effect to simulate movement through the consecutive exposure of discrete still images. A factor begins to play a crucial role, i.e. the speed at which the images change, or the number of image states per second that are necessary to create the illusion of continuous movement. It is the first understanding of the notion of "resolution", i.e. to what degree a movement has to be resolved into individual steps in order to appear continuous when presented in the zoetrope. Sixteen to eighteen images per second are sufficient to create the illusion of movement. The technical apparatus and the image sequence have to be matched and "synchronized " in order to simulate a movement that appears "natural" to the human eye. ${ }^{9}$

The introduction of image telegraphy and of television made it necessary to consider the aspect of resolution not only with regard to the segmentation of movement in time (temporal resolution, image frequency), but also with regard to the density of visual information on the image surface (image resolution). In order to transmit an image by means of electrical or electronic signals, the image has to be split up into a matrix of image points (pixels) that are more or less individually encoded into discrete signals, transmitted, and then decoded for display on a monitor with an equivalent pixel matrix. The higher the number of pixels and the greater the detail of the representation, the higher also the requirement of information that has to be coded and transmitted.

Here again, the human perceptual faculties play an important role, since the level of accuracy of the image representation depends on the accuracy of human vision and the degree to which adjoining elements of the image are perceived as separate, or seem to merge. Thus, in a fine matrix, different shades of gray can be constructed from different mixtures of white and black pixels, and on a color TV monitor, colors and shades are constructed from more or less bright pixels of red, green and blue. ${ }^{10}$

The physiological conditions of human vision also form a crucial factor in the construction of digitally coded images where, due to the limitations of transmission bandwidth, a balance has to be found between image accuracy and data volume. Image compression standards for still and video images, as well as for audio signals, seek to optimize this balance on the basis of "perceptual coding" that, like in the JPEG standard, explicitly takes the characteristics of human visual perception into account to mathematically model the optimal level of loss of detail for specific display environments. ${ }^{11}$

Artworks like Marcel Duchamp's Roto-Reliefs, or Jim Campbell's low-resolution video installations, or Thomas Ruff's jpeg photo series, not only articulate aspects of technical and human physiological dispositions - the visual media technologies on which they are founded already do that. As artworks, they reinvent the medium which they deploy and make its particular aesthetics available for further scrutiny. ${ }^{12}$ They are media for human perception. In so far as these works explore the articulation of human vision and technical media, they also highlight the entanglement of human perception in the technical set-up, and vice versa.

\subsection{Physical Conditions of Image-Making}

The classification of the conditions of image-making which I am suggesting here-physiological, physical, electronic, and algorithmic-points to the fact that images are not simply given, but that they always depend on highly specific and complex material conditions. The "media" which convey images never exist in a pure and definitive form but appear as phenomena and concepts resulting from such medial conditions.

A classification reaches its limits when the classes become so general that they have to cluster together absurdly different items. Only in contrast to the other classes of physiological conditions on one side, and electronic and algorithmic conditions on the other, does it make sense to group together image-making techniques that range from mechanics and optics, to chemistry.

Among the most explicit mechanical image machines are the Méta-Matics and "Drawing Machine" kinetic sculptures by Jean Tinguely. From the mid-1950s until the 1970s, Tinguely built a total of 17 sculptures, or apparatuses, with which drawings can be produced. Méta-Matic No. 8 (1958), for example, is a "table-top model" of $50 \mathrm{~cm}$ height and 110 $\mathrm{cm}$ width. It is a technical contraption on a tripod, with several flat sheet metal elements, circles, a rounded triangle, a spiral, which are painted black and attached to a support construction. An electrical motor is built into the pedestal on which the sculpture is placed, and is connected to the

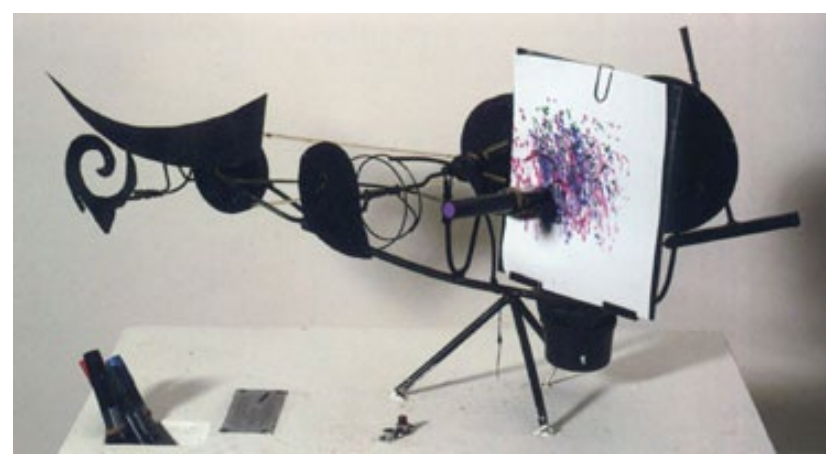

Jean Tinguely: Méta-Matic No. 8(1958), Moderna Museet, Stockholm. 
sculpture by a transmission belt. It drives a conveyor system which moves the different elements. In the right half of the sculpture is a vertically placed black metal sheet of $30 \times 20 \mathrm{~cm}$ to whose front a piece of A4 paper can be attached. A metal arm in front of this sheet can be equipped with a color pen. The arm is attached to one of the moving elements, and as the motor is switched on by means of a button in front of the sculpture, the entire construction begins to move and shake, the pen erratically hitting the sheet of paper in a radius of ca. $15 \mathrm{~cm}$. By applying pens of different colors in consecutive sessions, the sculpture can be made to produce polychromatic drawings.

In the series of drawing machines, Tinguely varied the construction in size and form. Some sculptures use human physical power, like the bicycle-driven Méta-Matic No. 6 (1959), but the general principle of a kinetic metal construction to which a pen and a piece of paper can be attached, remains the same. The sculptures are never anthropomorphic. The construction that holds and guides the pen determines the possible structuration of the drawing, the physical latitude of the construction and its actualization determine the graphic form. The act of drawing is an event that happens in time, and the duration of the event-timed by the duration of operating the mechanism-determines the density of the drawn color lines and strokes. Each drawing is unique.

What does it mean in this case to "make an image"? We are faced with a complex assemblage that encompasses the artist-engineer who constructed the machine; the machine with its particular physical structure that determines its operation and that includes both functional elements and "non-functional" elements which form part of the overall sculptural appearance and its performance; the visitor, user and operator who sets the process of drawing in motion; and the resulting drawing. Tinguely provides a deconstruction of the act of image-making-even though he reserved a special place for himself, given his awareness for the currency of the artist's name on the art market. The drawings were treated as artworks, they were signed and dated. ${ }^{13}$ In the context of the 1959 Paris art fair, Tinguely presented Méta-Matic No. 17, and at the same time he filed a patent for the principle of the drawing machine. He positions himself as both artist and inventor.

This setting, in which a material construction is left to operate according to its physical parameters framed by the laws of physics, is the basic principle of the physical conditions of image-making. They also come into play in the material practice of painting, or of image-making, by artists like Barnett Newman, Lucio Fontana, or Jackson Pollock.
Similarly, we can describe the operation of such physical affordances in the optical and photo-chemical explorations of László Moholy-Nagy, in the work of structural filmmakers like Hollis Frampton or Michael Snow, as well as in Tacita Dean's recent film-archaeological works. Such physical conditions and properties form a decisive part of the creation of artworks that receive a certain aesthetic "dowry" (Boehm) from them.

\subsection{Electronic Conditions of Image-Making}

The artistic exploration of the electronic conditions of image-making is closely tied to the development of television and video technologies in the 1960s and 1970s. There is little artistic engagement with electronics before 1960, and since the mid-1970s this engagement is more and more determined by digital systems which, even though they also rely on electronics for their signal processing, input and output devices, introduce another set of technical affordances to the creation of images.

Nam June Paik had used television sets in his exhibition Exposition of Music - Electronic Television (1963), manipulating the standard functionality of the electronic devices. The minimalist Zen for TV, for instance, which shows a single white line of pixels on the screen of a television set that is placed on its side, was the result of a technical manipulation of the vertical deflection plates in the Cathode Ray Tube of a television set, which is really not an electronic, but a physical, namely magnetic function of the CRT monitor. In 1968-1969, together with the electrical engineer Shuya Abe, Paik constructed the "Paik-Abe-Video-Synthesizer", one of the earliest examples of an electronic device that could not only manipulate recorded video images, but also create synthetic images.

A whole scene of artists, many of them in the USA, started in the late 1960s to explore the aesthetic potentials of electronic signal processing in video, first analogue, later with digital and hybrid analogue/digital systems ${ }^{14}$ Among them were Steina and Woody Vasulka whose work in the 1970s is exemplary for the spirit of experimentation and technical invention which dominated those years.

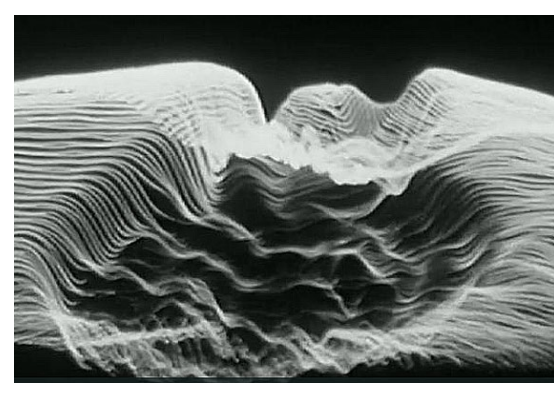

Woody Vasulka: Scan Processor Studies (1970s) 
The historical importance of these rather formalistic video artworks lies, firstly, in the exploration of an iconicity that is based on the given technical structure and specifications of the video system: an exploration that was pushed forward both by the invention of new technical tools that broadened the range of visual expression, and by the appropriation and transformation of existing technology. A generation later, Julien Maire's installation Exploding Camera (2005) is an example for work focused on the signal input of the video sensor. So between Maire, the Vasulkas, and the early Paik, we see that the video image can be tweaked and created at the three levels of input, signal processing, and output. Every electronic image is constructed from the technical components they explored.

\subsection{Algorithmic Conditions of Image-Making}

In a discussion of "the images of new media", Gottfried Boehm repeated, in 1999, the widespread assumption that digital images are "explications of calculation processes" which, in principle, "can take on any shape" and "resemble the programs on which they are based." 15 He claimed that "the new media are literally 'media', i.e. the conditions of possible images," and that it is questionable "whether and how the new media are able to achieve genuine iconic manifestations."16

However, iconicity is always based on technical conditions, and it is an arbitrary decision by Boehm to use some technical conditions (like the material conditions of painting) for defining a standard of iconicity. Rather, the phenomenological definition of the image that Boehm also generally adheres to has to be seen as independent of specific techniques. At the same time, in order to be able to describe an image and to understand its aesthetics, it is necessary to be able to analyze both its phenomenological and its technical aspects. According to Boehm, "in the sense of iconic valences, [the images of new media] are not images at all, but simulations." ${ }^{17}$ Yet, this can also be said of the "images of old media", whose materiality of oil paint and linen canvas or wood "simulate" an icon, rather than being one.

Like the previous sections, our treatment here of the algorithmic conditions of image-making can only hint at the complexity of these conditions, with the aim of underlining the "iconic valences" of specific techniques and their applications.

The pioneers of computer graphics, like Georg Nees, Frieder Nake, or Michael Noll, used the potential of computer algorithms to describe graphic structures which could be printed, for instance, on a plotter. Changing and elaborating the computer code made it possible to produce new and more complex graphic structures. This dual structure of a text-based, algorithmic computer code that is rendered as a graphic output, is at the heart of the dispute about the iconic status of "computer images." The range of graphic expression in the arts has been greatly extended since the first experiments in the 1960s, for instance in the multi-dimensional mathematics of Manfred Mohr's images, and the continuously changing and evolving generative computer graphics of artists like Casey Reas have created a new genre of images altogether. Yet another aspect is added by interactive computer graphics, like those of Golan Levin, which offer the rendered images for manipulation and transformation to the viewer. Such images need to be understood as technical constructs, and they afford an extended definition of what constitutes an image. Always the aesthetics of such an image is related to the manual or algorithmic execution of the rules, or intentions, and with the respective output medium of canvas, a plotter or printer, or monitor.

A second field where the algorithmic conditions of image-making are important is that of digital video. By means of digital techniques, both optically produced images and those that are the result of electronic or digital image synthesis, can be processed and the iconic vocabulary extended through editing, layering, compositing, time-axis manipulation, etc. ${ }^{18}$ And a third field of image-making, in which algorithms and their affordances play a significant role, is that of Internet-based art. Since the early 1990s, the Internet has created an evolving technical environment in which new forms of communication and interaction have taken shape. The communication protocols of the Internet-like the coding of digital video-are algorithmic on one substantial level, even though this digital layer merely provides the rules and information processing according to which electronic signals are then used to switch and transmit what is to be encoded and decoded at the "terminal“ ends. ${ }^{19}$

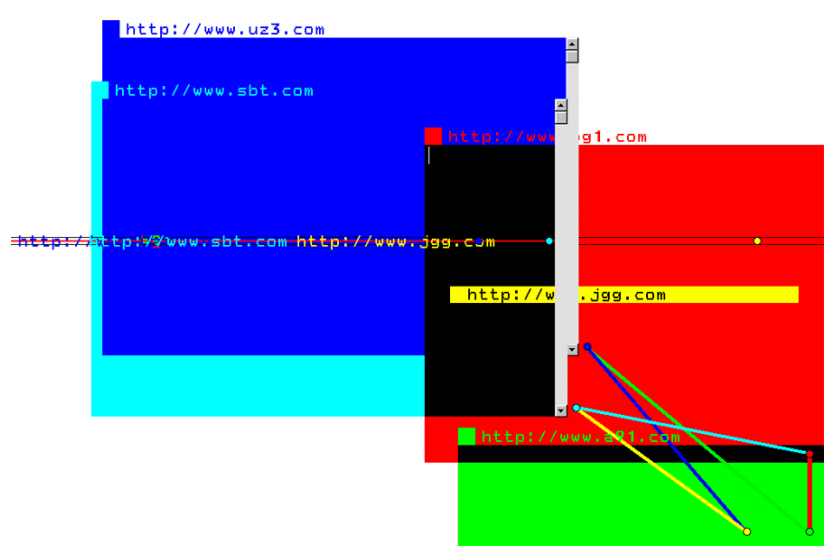

JODI: Wrong Browser (2000) 
Inge Hinterwaldner has discussed this type of Internet-based artistic work with regard to theories of the image and interactivity. ${ }^{20}$ She analyzed aesthetic strategies like „iconoclasm, minimalism, negation, fragmentation, decomposition, dys- and refunctionalization, irritation, censorship, etc., ${ }^{21}$ with regard to the question, „whether everything that a computer screen shows, is an image." ${ }^{22}$ Does the modernist criterion of the self-questioning of an artistic medium apply in the case of the computer, given the "lacking difference between medium and image "23? Hinterwaldner suggests not to posit the technical support of the screen as an absolute, but to "bear in mind the specific iconic qualities, and to pay attention to the situative constellation of medium, context, reception, intention and motif." ${ }^{24}$ In a software-based environment like the Internet, the image and its "staging" can be programmed in the same medium, offering the "possibility of interlacing iconicity with symbolic and programmed actions." ${ }^{25}$ An important realization that Hinterwaldner also points towards is that images like those of JODI and other Internet artists, do not exist in one medium, but are always connected in a chain, or cascade, of different media with their respective technical and aesthetic affordances and conditions, which means that, for instance, there is no physical image that is not also taken in by physiological perception, and there is no algorithmically constructed image that does not also have electronic, and physical, and physiological aspects. The operation of certain functions, of switches, mirrors, LEDs, etc., is electronically or digitally controlled at different levels of size and speed, but in the end there are physical and optical occurrences which make an image, while digital and electronic technologies form part of the systemic conditions and determinants under which that production, and their reception, can take place. ${ }^{26}$

\section{CONCLUSION}

If we consider the materiality and the mediality of images in this way, it becomes possible to open up Gottfried Boehm's understanding of the image and of "iconic difference" to a technically differentiated understanding of image-making. As early as 1996, Boehm writes: "It would certainly be inappropriate and against the spirit of hermeneutic distinction if one were to treat all technical images equally, criticizing them as deficient. The artistic possibilities of photography (and other image technologies) are obvious. They are able to do what is attributed to all art: opening up new views onto reality, which wouldn't exist without them." ${ }^{27}$
We should reject any distinction between "technical" and "traditional" images. Anette Hüsch and others have argued against the myth of a stable and "traditional" image order, because even photography, that supposedly "objective" image medium, has, from its very introduction onwards, been diagnosed as epistemologically unstable. ${ }^{28}$ Also, imaging techniques such as central perspective, camera obscura and the pantograph, all used by painters and image-makers well before the introduction of photography, suggest that it is impossible to draw a clear distinction between "technical" and "non-technical" images. A thorough understanding of the cultural techniques of electronic and digital image production can bring "stable ground" 29 to the analysis of contemporary image economies. Every epoch has its "conventional" images, and time and again these conventions change and expand.-And there will, no doubt, be a generation of art historians and visual studies scholars who will look at Arduino boards, the DICOM standard, and at DLP-projectors with the technical competency necessary to redefine the concept of the image.

Julien Maire's title for the installation Memory Cone explicitly relates to Henri Bergson's concept of memory and the "memory cone" that articulates the expanse of all our memories, dispersed across the recesses of our mind-represented by the base of the "cone", with what we remember at a specific moment, literally pinpointed by the tip of the cone. ${ }^{30}$ Yet, Maire does more than-as Edwin Carels has suggested - offer a "translation of Bergson's metaphor into an optical process". ${ }^{31}$ The technical contraption that Maire has built constructs a setting and a form of relation between images and actions that is unique and allows for an aesthetic experience that cannot be had otherwise. In that sense, Maire's Memory Cone surpasses Bergson's conception and offers its own model experience for the way in which we interact with our memories, as well as with technically mediated images. This model is made possible by the specific technical construction, and it inherits the conceptual hybridity of the technical, visual and interactive elements of the installation. In the same way that Bergson used "cinema as a metaphor for the mechanism of our thinking " ${ }^{\prime \prime}$, Maire uses the philosophical concept of the memory cone as a metaphor for the mechanisms of image construction. 


\section{ENDNOTES}

1. Cf. Cubitt, 2014, for a comprehensive historical study of the techniques of image making.

2. Cf. Broeckmann, 2016, chapter 4, “Image Machine", for a more in-depth discussion of the question posed here.

3. Cf. Carels 2012, p. 187.-The article by Edwin Carels (2012) forms a first comprehensive attempt to discuss Maire's work in a media-theoretical context, giving less attention to its artistic aspects, and presenting the works as media-archaeological projects rather than artworks in their own right.

4. Boehm 1999, p 173, transl. AB. (... als dessen minimales Definiens wir die gleichzeitige Wahrnehmbarkeit von Darstellungsebene und Dargestelltem, von medialer Prämisse und ikonischer Formung nennen können.)

5. Cf. Boehm 1999, p. 173-174.

6. Cf. Boehm 1999, p. 170.

7. For a commentary on Crary's theses, cf. Kittler 2010. For studies of the different media apparatuses developed during the 19th century, cf. Dewitz 2002, Huhtamo 2013.

8. Cf. Krauss 1993, p. 95-103, among others for a rendering of the erotic associations some of the disks bring up.

9. The stroboscopic effect is of course also crucial for the development of film.-Examples of contemporary zoetropic artworks are Gregory Barsamian: The Juggler, 1996; Toshio Iwai: Time Stratum II, 1985; and Julien Maire: Les Instantanés, 1998.

10. For a summary of the technical development of image resolution, cf. Kittler 2010. Contemporary artistic examples that explore resolution are Jim Campbell: Running and Falling, 2004, and Scattered Light, 2010; Julien Maire: Low Resolution Cinema, 2005; Aram Bartholl: 0,16, 2009.

11. Cf. Alan C. Bovik: The Essential Guide to Image Processing. Amsterdam, 2009; Mohammed Ghanbari: Standard Codecs: Image Processing to Advanced Video Coding. London, 2003. On digital video codecs, cf. McKenzie 2008.—Cf. Thomas Ruff: jpegs. Cologne: DuMont, 2009.

12. Cf. Krauss 2011 on the trope of the "reinvention of the medium" which can, I believe, be extended to works like those of Maire, Campbell, JODI, and others.
13. The titles of Tinguely's machines are not all systematic, but typical for the pre-1970 phase is, for instance: "Painting by Méta-Matic No. 20 in collaboration with Klara Hulten." During a public session with Méta-Matic No. 8 in 1974, the paper was stamped, dated and signed with the operator's name on the reverse side, and signed by Tinguely on the front side.

14. Cf. Spielmann 2008.

15. Boehm 1999, p. 175.

16. Boehm 1999, p. 175-176. He hints that the work of Gary Hill "leaves no doubt" that this potential is principally given (p. 176)

17. Boehm 1999, p. 176.

18. Cf. for instance Zbigniew Rybczynski (2011), video installations and performances by the group Granular Synthesis, or works by Tania Ruiz Gutierrez.

19. Cf. for example the work of JODI (Joan Heemskerk, Dirk Paesmans)

20. Cf. Hinterwaldner 2006.

21. Cf. Hinterwaldner 2006, p. 355.

22. Cf. Hinterwaldner 2006, p. 356 (ob alles Bild ist, was der Bildschirm zeigt).

23. Cf. Hinterwaldner 2006, p. 356.

24. Cf. Hinterwaldner 2006, p. 357.

25. Cf. Hinterwaldner 2006, p. 374.

26. Notably, media-archaeological practice in contemporary art (Julien Maire, Paul DeMarinis, Joost Rekveld, Gebhard Sengmüller, Tacita Dean) is, currently, mainly oriented at pre-electronic media techniques, although its principles can also be applied to electronic and digital media techniques (as demonstrated by the revival of 8-bit aesthetics, esp. in relation to game culture).

27. Boehm 2007, p. 246.

28. Cf. Hüsch 2006.

29. A criterion formulated by Boehm (2007, p. 248).

30. This relationship has been discussed by film and media historian Edwin Carels who has also pointed to the technical aspects of 
duration and memory, terms which are crucial for both Maire and Bergson. Cf. Carels 2012, p. 187-188.

31. Carels 2012, p. 187.

32. Carels 2012, p. 188.

\section{BIBLIOGRAPHY}

Boehm, G. (1999). Vom Medium zum Bild. In: Y. Spielmann, G. Winter (Eds.), Bild-Medium-Kunst (pp. 165-177). München: Fink.

Boehm, G. (2007). Zuwachs an Sein: Hermeneutische Reflexion und bildende Kunst. In: G. Boehm: Wie Bilder Sinn erzeugen: Die Macht des Zeigens (pp. 243-267). Berlin: Berlin University Press. Original publication, 1996.

Broeckmann, A. (2016). Machine art in the twentieth century. Cambridge, MA: MIT Press.

Carels, E. (2012). The productivity of the prototype: on Julien Maire's cinema of contraptions. In Vanderbeeken, Robrecht, Stalpaert, C., Depestel, D., \& Debackere, B. (Eds): Bastard or playmate? Adapting theatre, mutating media and contemporary performing arts (pp.178-192). Amsterdam: Amsterdam University Press.

Cubitt, S. (2014). The practice of light: a genealogy of visual technologies from prints to pixels. Cambridge, MA: MIT Press.

Dewitz, B. (2002). Ich sehe was, was du nicht siehst. Sehmaschinen und Bilderwelten. Die Sammlung Werner Nekes. München: Steidl.

Hinterwaldner, I. (2006). When windows attack ... doors won't help. Zur Hinterfragung von Bildlichkeit und Interaktivität in der Netzkunst. In B. Mersmann, M. Schulz (Eds.): Kulturen des Bildes (pp. 355-374). München, Fink.

Hüsch, A. (2006. Ein Relikt vergangener Zeiten. Anmerkungen zum Begriff des traditionellen Bildes. In B. Mersmann, M. Schulz (Eds.): Kulturen des Bildes (pp. 345-353). München, Fink.

Huhtamo, E. (2013). Illusions in motion. Media archaeology of the moving panorama and related spectacles. Cambridge, MA: MIT Press.

Kittler, F. (2010). Optical media. London: Polity.

Krauss, R. (1993). The optical unconscious. Cambridge, MA: MIT Press.

Krauss, R. (2011). Under blue cup. Cambridge, MA: MIT Press.

Rybczynski, Z. (2011). Traktat über das Bild. (2009) In S. Zielinski, P. Weibel (Eds.): Der Stand der Bilder. Die Medienpioniere Zbigniew Rybczynski und Gabor Body (pp. 42-55). Nürnberg: Verlag für Moderne Kunst.

Spielmann, Y. (2008): Video. The reflexive medium. Cambridge MA: MIT Press.
CORRESPONDENCE

Andreas Broeckmann

Scharnhorststraße 1

21335 Lüneburg

Germany

E-mail: broeckmann@leuphana.de

Published online, 2017

ISSN 1749-3463 print/ISSN 1749-3471

https://doi.org/10.14434/artifact.v4i1.13125

(C) 2017 Artifact 